\title{
Antiretroviral Drug Therapy in Benue State, Nigeria: A Follow-up of 20 PLWA in a Private Hospital in Gboko
}

\author{
Fred Iornongo Tamen ${ }^{1 *}$, John Kpamor ${ }^{1}$, Nyitor Alexander Shenge ${ }^{2}$ and Terzungwe Ayebe ${ }^{1}$ \\ 1. Department of Psychology, Benue State University, Makurdi, Nigeria \\ "E-mail: ftamen@yahoo.co.uk \\ 2. Department of Psychology, University of Ibadan, Ibadan, Nigeria \\ ${ }^{2}$ E-mail: alex71851@yahoo.com
}

KEYWORDS Antiretroviral. Drug. AIDS. Benue. Nigeria. Follow-Up

\begin{abstract}
The need for a drug policy on HIV/AIDS was investigated through a pilot clinical study of 20 Persons Living With AIDS (PLWA), (8 males and 12 females) who took combivir antiretroviral drugs (ARV). Their age range was between 11 and 60 years with those between 11 and 40 years presenting most for the drug therapy. Each participant responded orally or in writing to a self-developed questionnaire. Hospital case record files were also reviewed for more data to establish additional complications during therapeutic interventions with combivir therapy. Recommendations for commencing ARV drug therapy as well as side effects and opportunistic infections for combination drug therapy were made. These have significant implications for HIV/AIDS victims, and patients on ARV drugs, counselling and psychotherapy. Policy recommendations emphasise need for drug in terms of combination therapy, sustained health education, empowerment and capacity building, expanded facilities and above all, increase in uptake and availability of ARV drugs.
\end{abstract}

\section{INTRODUCTION}

Not much work has been done on antiretroviral (ARV) therapy in Nigeria until recently. This can be attributed to the poor coordination efforts in the fight against human immunodeficiency virus (HIV). In 1984, it became clear that HIV was the known cause of acquired immunodeficiency syndrome (AIDS). Not quite long after this knowledge, drugs that could devastate the virus were placed on clinical trails. In 1985, azidothymdine (AZT) became the first ARV drug to be tried on humans.Enthusiasm over the promising results of AZT quickly waned because within eight years, it became "known that AZT's modest benefits typically faded within a year, probably because the virus quickly mutates into new forms that are resistant to the drug's actions" (Cohen, 1993).

There are currently more ARV drugs and their clinical effectiveness has improved remarkably. Fischl (1990) explains that drug development for anti-HIV therapies rests on our knowledge of replication cycle of the virus. Thus, some sustained inhibition of viral replication might lead to partial reconstruction of the immune system and a substantial reduction in the risk of clinical disease progression. Most importantly, blocking the initial phases of viral replication prevents infection of new cells.

Two groups of antiretroviral compounds that interfere with HIV reproduction are reverse transcriptase inhibitors and protease inhibitors. Retroviruses derive their name from the fact that their genomes encode a peculiar enzyme, reverse transcriptase which permits deoxyribonucleic acid (DNA) to be transcribed from ribonucleic acid (RNA). This allows HIV to produce copies of its own genome as DNA in human host cells such as the CD4 helper lymphocyte (Mortimer and Loveday, 2001). Thereafter viral DNA nuclear material become incorporate in the lymphocyte genome to begin chronic HIV/AIDS infection.

The best known of the antiretroviral compounds are the reverse transcriptase's dideoxynucleosides. They mechanically exert anti-HIV activity at the reverse transcriptase level. First, they compete with essential cellular substrates for the formation of proviral DNA by reverse transcriptase. Second, they act as chain terminators when proviral DNA is synthesized (Fischl, 1990). Third, they inhibit the reverse transcriptase enzyme that convert viral RNA to DNA which is then integrated into the nuclear substrate of human cells for replication. Examples of drug agents from this compound include zidovudine (Retrovir) dideoxycytidine (ddC), dideoxyinosine (ddI), didehydro - dideeoxythymidine (D4T), and phosphonoformate (Foscarnet).

The protease inhibitors decrease activities of the protein producing protease enzyme which covers the viral genome body. Once the genomic 
material is left without a membrane and unprotected, it is destroyed. (Hasslett et al., 1999). Some of these drugs are Sequinavir, Ritonavir and Indinavir.

Major ARV drugs that found their way into the Nigerian market shortly after the Federal Government passed a vote of he confidence on locally made anti-HIV drugs in 2001 are: zidovudine (AZT), stavudine (D4T), lamivudine, nelfinavir, saquinavir, Ritonavir, Videx, Trizivir, and Combivir.

The HIV life cycle consists of nearly 20 steps. Any interruption at any step could potentially halt the virus from replication. Most clinical successes in the past were focused on crippling the virus at the point where its genetic material (RNA) undergoes reverse transcription into DNA. Other equally promising investigative methods have aimed at terminating the HIV life cycle. For instance Cohen (1993) reports that a regulatory protein called Tat boosts the transcription of latent HIV DNA in a gene into messenger RNA (MRNA). Consequently, anti-Tat drug is undergoing trial in infected people living with replication in both acute and chronic HIV infected cells. Tat is essential for $\mathrm{HIV}$ replication in cultured cells. It is also a positive transactivator that stimulates transcription and translation of ribosomes into budding new virus particles (Johnston et al., 2001).

A similar regulatory genetic protein called Rev assist Tat to more RNA around a cell. Since Rev assist HIV's replication more than Tat, its blockage offers potentials for interrupting viral reproduction even far more than that of anti-Tat agents. Many researchers see Rev as best genetic target for the virus. However, other researchers also think that a gene therapy approach that focuses one or two viral proteins would still end up as a fatal error. Be that as it may, there is a need to investigate the clinical usefulness of combining anti-Tat and anti-Rev agents with other anti-retroviral compounds.

Mortimer and Loveday (2001) note that modern treatments are using combinations of nucleoside analogues and protease inhibitors to change the prognosis for HIV carriers. In most cases, appreciable drop in virus load in the blood is sustained and the CD4 lymphocyte count is returned to almost it normal levels.

\section{DRUG THERAPY}

The two classes of anti-retroviral drugs (protease inhibitors and reverse transcriptase) could be prescribed preferably in combination to avoid resistance; and promote synergistic, antagonistic and additive effects. Controlled studies have shown improved prognosis and survival with combinations of 2 or 3 ARV agents taken together (Spector et al., 1989). Infact many drug combinations are effective and monotherapy is no more in vogue. In very severe HIV infections, triple or cocktail therapy is most effective.

Tuder-Williams and Gibb (2002) reports that highly encouraging results have come from triple or quadruple drug combinations in selected infants. Thus, the use of highly activated antiretroviral therapy (HAART) enables viral load to fall substantially.

Combination therapies have enjoyed a long and successful antecedence. For instance, in bacterial, fungal and viral infections, combination microbial therapy and cancer chemotherapy have been the treatment of choice.

Zidovudine was the first agent to be used as a reverse transcriptasse inhibitor. Although it has proven effective and efficacious in HIV treatment, its limitations in terms of disease progression, toxicity and adverse side effects have been noticed. It also loses its efficacy as an anti-HIV agent due to the emergence of resistance from the human host cells.

In this study, combivir was selected for clinical investigation. It is a combination of Zidovudine (300 $\mathrm{mg}$ ) and Lamivudine (150 mg). Zidovudine is a deoxythymidine analogue that is well absorbed from the gut. It is toxic to patients with hepatic insufficiency. Zidovudine has been shown to decrease the rate of clinical progression and prolong survival in HIV patients. The most common adverse effect of Zidovudine is myclosuppression a1 intolerance, headaches, insomnia, hyperpigmentation of nails and myopathy.

Lamivudine is a cytosine analogue with activity against HIV-1. Its potential side effects are headaches, insomnia, fatigue and a1 discomfort. It develops synergistic action with a variety of antiretroviral nucleoside analogues. It is hoped that its combination with other effective drugs might forestall the development clinically significant resistance (Kutzang et al., 2001).

Drugs like nevirapine, stavudine and ritonavir are more toxic. Mitochondrial toxicity is suspected to produce cumulative side effects from them. The most severe adverse effect is lactic acidosis which is responsible for a number of HIV deaths.

Many therapy failures have been traced to 
drug failures. The South African HIV Clinician Society (SAHCS) reports that no currently available antiretroviral drug is sufficiently potent to provide HIV suppression which occurs faster with 5 different AIDS drugs. It however noted that the synergistic use of 3 agents remains the standard of care, and produces a potent and sustained reduction of viral load (Sofola, 2000).

Antiretroviral drugs are not curative. They mainly prevent the virus from developing into AIDS. So people with HIV are required to take them for an indefinite period of time, possibly for the rest of their lives.

In a country like Nigeria with a collapsed health care system, the administration of these drugs is "unsafe". For instance, Nigerians taking the drug will have the problem of irregular check-up because only few health institutions in the country can conduct such tests. The National Institute for Medical Research (NIMR) and the National Institute of Pharmaceutical Research and Development (NIPRD) have virology laboratories that carryout CD4 counts and viral load count. They are institutions that can provide the needed technical sophistication and scientific analysis for treatment, prevention and care measures to be carried out.

The efficacy of other drugs has been put to the test. They do not eliminate the viral load completely. When a drug's administration is discontinued, the viral load rebounds and swells up in number. Besides, the efficacy of the drug may be worsened due to toxicity, poor compliance and cost of treatment. Fischl (1990) relates HIV treatment reduces individual drug doses, delays development of resistance or low sensitivity and increases drug effectiveness.

Bt regrettably, HIV has been known to mutate into new strains that defile every other ARV drug trials or combinations. The fact that so much type can co-exist in a single episode indicates a crucial need to bombard the virus with several drugs at one shot - the shot gun approach - so as to beat its rate of mutation.

There is at present no clearly marked pathway for this combination therapy and so the search for the right combination persists. Overall, it is directed at arresting different stages of the virus' life cycle and thwarting its replication rates.

In Western countries, there are various guidelines for AVR therapy for adults and children. Those for adults are taken from Weller and Williams (2001) and presented in Table 1.
Table 1: Recommendation for starting ARV therapy

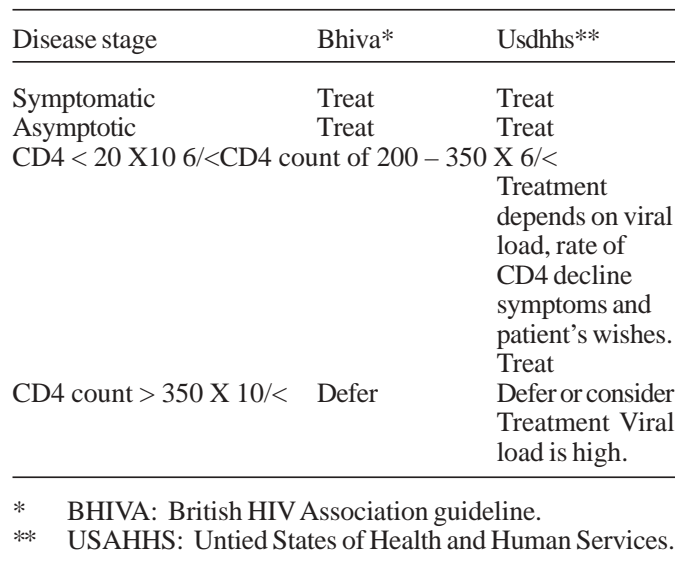

The optional time to initiate therapy with current ARV drugs has not been established in clinical studies. CD4 count pleama viral load are predictors of the estimated risk of progression of AIDS. These are a determining factor in starting AVR drug therapy.

Determination of the CD4 count and viral load is difficult in Nigeria. So a more practical approach should be taken to determine when to start therapy and the choice of therapy. Such factors include:

Risk of clinical disease progression

Willingness of patient to start therapy

Clinical manifestation of combination regimen

Ability and motivation of patients to adhere to therapy

Drug toxicity profile

Pill burden and dosing schedule

Future drug options

Likelihood of drug resistance

Drug - drug interactions.

In starting therapy with AVR drugs, the patient's clinical history should be taken, and physical and mental examinations performed laboratory assessments such as haemogram urinalysis, liver function tests and chest $\mathrm{X}$-rays should be done and found not to be severely impaired.

Apart from high cost in Nigeria, the ARV drugs have many side effects, which could ultimately affect drug compliance. SAHCS documented the major side effect of all existing ARV drugs. They include myclosuppression, gastrointestinal intolerance, pancreatities, peripheral neuropathy, allergic reaction, hypertrophy, lactic acidosis, 
raise blood cholesterol and triglycerides, insulin resistance and neuropsychiatric manifestations (Sofola, 2000).

In Nigeria, many side effects have been associated with ARV therapy and they include development of pedal Oedema, respiratory distress, abdominal pains, digestive disorders and drug fever after ingestion of combivir. However a priority with have to be developed by using rational and effective regimens, minimi-zing toxicity and resistance; and above all maximizing adherence to combination therapy for satisfactory outcomes.

\section{METHOD}

This study is a pilot study of 20 patients at Sayco Hospital, Gboko South. The sample was restricted to 20 because of the high cost of the Antiretroviral drugs. The drugs are being supplied by private sources. Only those that could afford an initial deposit of $\$ 60,000$ were allowed to take part in the study. The selection of the sample was based on first come, first served. Hence, the first twenty to register were used in the clinical study. Only one antiretroviral drug instead of a cocktail was used on the clinical sample because they could not afford the costs of a cocktail therapy.

Instruments: A self-developed questionnaire was given to each respondent to fill out. Those who could not write were interviewed orally in the local language with the same translated questions.

Their case record cards were also reviewed to determine presence of complications and reasons for stopping therapy, especially where the patients did not know the answers.

From these 2 sources information was gathered and analyzed.

\section{RESULTS}

From the age distribution shown in Table 2, we can see that the age of eleven to forty years are the most who presented for HIV/AIDS antiretroviral drug therapy. This confirms the findings in literature which says that those in these age brackets are the most sexually active. It could also mean that women in the study sample had more economic support to afford the initial deposit. Haslet et al. (1999) maintained that the economic and demographic impact of HIV infection is profound and the disease is prevalent between the ages of $15-50$ years of age.

Table 2: Age distribution of patients on Combivir

\begin{tabular}{lrc}
\hline Age & No. & $\%$ \\
\hline $0-$ 10years & Nil & 0 \\
$11-$ 20years & 2 & $10 \%$ \\
$21-$ 30years & 8 & $40 \%$ \\
$31-$ 40years & 8 & $40 \%$ \\
$41-$ 50years & 1 & $5 \%$ \\
$51-60$ years & 1 & $5 \%$ \\
\hline Total & 20 & $100 \%$ \\
\hline
\end{tabular}

Although the sample selection appeared biased in favour of women, it can be seen in Table 3 that since more women first reported for the study, it could be explained in terms of females being more concerned with their health.

Table 3: The sex distribution of patients

\begin{tabular}{lrr}
\hline Sex & No. & $\%$ \\
\hline Males & 8 & $40 \%$ \\
Females & 12 & $60 \%$ \\
\hline Total & 20 & $100 \%$ \\
\hline
\end{tabular}

Although it is said that HIV has no educational barriers, majority of those affected that reported for the initial pilot antiretroviral drug therapy were secondary school level, followed by those with University education (Table 4).

Table 4: Educational qualification of the patients on Combivir

\begin{tabular}{lrc}
\hline Education & No. & $\%$ \\
\hline No Education & 1 & $5 \%$ \\
Primary school & 0 & 0 \\
Secondary school & 10 & $50 \%$ \\
University & 8 & $40 \%$ \\
Postgraduate & 1 & $5 \%$ \\
\hline Total & 20 & $100 \%$ \\
\hline
\end{tabular}

It can be seen from Table 5 that majority of those who could afford the initial deposit and who first presented themselves for therapy were business men and women followed by civil servants.

Only those who earn higher income can afford treatment with combivir due to the high cost. It costs an average of N45, 000 monthly. This agrees with the fact that "about $90 \%$ of cases in developing countries are unable to afford the 
Table 5: The occupational distribution of patients

\begin{tabular}{lrr}
\hline Occupation & No. & $\%$ \\
\hline Businessmen/women & 15 & $75 \%$ \\
Civil servants & 4 & $20 \%$ \\
Drivers & 1 & $5 \%$ \\
\hline Total & 20 & $100 \%$ \\
\hline
\end{tabular}

expensive medical care required..." (Haslett et al., 1999).

Patients started taking drugs within 1 year of being diagnosed with HIV/AIDS. This shows they are quite eager to start therapy. Those delaying beyond 12 months ascribed it to time needed to save some money to start combivir therapy (Table $6)$.

Table 6: The time lag between diagnosis and start of Combivir therapy

\begin{tabular}{lrr}
\hline Time & No. & $\%$ \\
\hline $0-12$ months & 10 & $50 \%$ \\
$13-24$ months & 5 & $25 \%$ \\
$25-36$ months & 5 & $25 \%$ \\
$36-$ above & 0 & $0 \%$ \\
\hline Total & 20 & $100 \%$ \\
\hline
\end{tabular}

Majority of the respondents (60\%) believed strongly that combivir can cure AIDS completely (Table 7). Patients were not given adequate information as regards the ability of the drug to cure HIV/AIDS.

Table 7: Patients' perception of Combivir efficacy

\begin{tabular}{lrc}
\hline & No. & $\%$ \\
\hline Yes & 12 & $60 \%$ \\
No & 5 & $25 \%$ \\
Undecided & 3 & $15 \%$ \\
\hline Total & 20 & $100 \%$ \\
\hline
\end{tabular}

Few patients had CD- cell count. None had a Liver Function Test (LFT) due to lack of equipment. The average Pcv before therapy should be $30 \%$ and WBC count above $2500 \mathrm{C} /$ $\mathrm{mm}^{3}$. No platelet count was done due to lack of adequate personnel and equipment etc. for starting ARV drug therapy.

These tests are important because Combivir can alter liver function, cause myelodepprression leading to neutropaenia and anemia (Glaxowellcome drug literature). The virology laboratories include National Institute for Pharmaceutical Research and Development (NIPRD) and National Institute for Medical
Research (NIMR) which reportedly do CD4 and viral load counts and provide the needed sophisticated technical and scientific analysis. The analysis allowed treatment and cure measures to be carried out. The inadequacy of these tests is a far cry from an ideal situation. In this hospital, and in many others in Nigeria patients undergo combivir therapy without adequate baseline test and without adequate monitoring. This has a lot of implications for the side effects experienced by patients.

Table 8: Investigations carried out before beginning Combivir. The following investigations are necessary before Combivir therapy must commence. They include

\begin{tabular}{lc}
\hline Investgation & No. \\
\hline Liver function test & Nil \\
$\mathrm{CD}_{4}$ count & Nil \\
X-ray & 5 \\
VBC and differential & 20 \\
Platelet count & Nil \\
Pcv & 20 \\
Viral count & Nil \\
\hline
\end{tabular}

Table 9: Recommendations for starting antiretroviral drug therapy

\begin{tabular}{lll}
\hline Disease stage & BHIVA & USDHHS \\
\hline Symptomatic Asymptomatic & Treat & Treat \\
CD4 $<200 \times 10^{6 / L}$ & Treat & Treat \\
CD4 $200-$ & Consider & \\
$350 \times 10^{6 / L}$ & treatment if & \\
& $\begin{array}{l}\text { CD4 declines } \\
\text { symptoms and }\end{array}$ \\
& wishes & Treatment \\
CD4 $>350 \times 10^{6 / L}$ & Defer & $\begin{array}{l}\text { Treatment of } \\
\text { viral load is } \\
\end{array}$ \\
& & high \\
& & high \\
& &
\end{tabular}

1. BHIVA: British HIV Association guidelines

2. USDHHS: US Department of Health and Human Services.

These array of side effects are expected especially nausea, headaches, vomiting, loss of weight, abdominal symptoms, Pedal oedema, depressive disorders and anorexia (Table 10). The Southern Africa HIV Clinician Society documented the major side effect of the ARV drugs to include myelosuppression, gastrointestinal intolerance, pancreatitis, peripheral neuropathy, allergy, lactic acidoxis, lipodistrophy, hypercholes-terolanaemia and neuropsychiatric manifestations. Here, apart from clinical signs and symptoms, the lack of adequate facilities means that many of these side effects remain undetected during therapy. 
Table 10: Various side effects reported while taking Combivir drug therapy

\begin{tabular}{lrr}
\hline Side effects & No. & $\%$ \\
\hline Headaches & 20 & $100 \%$ \\
Nausea & 20 & $100 \%$ \\
Vomiting & 15 & $75 \%$ \\
Dizziness & 4 & $20 \%$ \\
Weight loss & 20 & $100 \%$ \\
Dyspnoea (Difficulty in breathing) & 2 & $10 \%$ \\
Oedemia (body swelling) & Nil & $0 \%$ \\
Anemia (shortness of red blood cells) & 4 & $20 \%$ \\
\hline
\end{tabular}

It appears that even though patients are undergoing therapy, there is still marked immunosuppression, thus allowing for opportunistic infections to occur. This agrees with the warning that "cambivir is not a cure for HIV infection and patients may continue to experience illnesses associated with HIV infections including opportunistic infections".

Table 11: Opportunistic infections noticed in patients taking Combivir drug therapy

\begin{tabular}{lrc}
\hline Infection & No. & $\%$ \\
\hline Oral thrush & 5 & $25 \%$ \\
Tuberculosis & 3 & $15 \%$ \\
Diarrhea & 15 & $75 \%$ \\
Genital ulcers & 5 & $25 \%$ \\
Chronic fever & 20 & $100 \%$ \\
\hline
\end{tabular}

The Average Period of Drug Ingestion : This was on average 12 months. Note that this is a drug they are supposed to take for life. For them to have taken it for an average of only 12 months portends a lot of implications for anti retroviral drug therapy.

It can be seen from Table 12 that those who interrupted the therapy due to financial reasons were $7(35 \%)$. Those interrupting because of severe side effects followed this, which constituted 3 $(15 \%)$. The remaining $2(10 \%)$ of the respondents did so on the advice of the medical doctor.

Although other factors such as illness and severe side effects can interrupt therapy. Lack of money is the greatest challenge facing those on combivir. At a cost of N50, 000 - N60, 000 per month in Benue State it would appear too much money for many to afford. In contrast, in Lagos, patients are relatively well to do economically. The side effects rather than monetary constraints were found to be responsible for the patients stopping or interrupting therapy. Benue State is an agrarian state and only subsistence farming is practised on a large scale. Commercial activities are at best very low resulting in poor pay of her citizens. Although the majority of our pilot sample appear to be only those from the commercial sector, at the moment, it seems they are the only ones who could afford the cost of treatment.

Table 12: Interruptions or stoppage of Combivir drug therapy.

\begin{tabular}{lcc}
\hline & No. & $\%$ \\
\hline Severe side effects & 3 & $15 \%$ \\
Doctors advise & 2 & $10 \%$ \\
Financial & 7 & $35 \%$ \\
Unavailable & 0 & $0 \%$ \\
\hline Total & 12 & $60 \%$ \\
\hline
\end{tabular}

\section{CONCLUSION}

This study is significant in that though patients are willing to take antiretroviral drugs, they lack the necessary financial resources for expensive laboratory testing and purchase of drugs. Other reasons such as severe side effects and illness (Opportunistic Infections) can interfere with continuing drug therapy for life as required. The study is also significant because the side effects experienced by patients has a lot of implications for counseling and psychotherapy of people living with HIV/AIDS.

\section{RECOMMENDATIONS}

From the above conclusion, the recommendations to be made include that the persons suffering from this malady should be adequately educated on the use of antiretroviral drugs. There is need by government, NGOs and private spirited individuals/philanthropists to build the capacity of health personnel so as to be able to know exactly how to monitor patients and to detect their side effects as well as how to manage these side effects.

The government should provide adequate facilities for virological studies and monitoring of patients. At the moment, CD4 count is only done at the Federal Medical Centre in the whole of Benue State.

There is need to educate the patients to know what drugs they are using, the side effects, what to do when they have the side effects and to enhance compliance.

The role of government in importing the drugs should be encouraged so that cheap, affordable 
and authentic drugs are available to all patients. They should not only be in designated institutions as it is the current practice. Private, nongovernmental institutions and mission hospitals should be involved in ARV drug therapy.

Patients and their communities need economic empowerment to improve their purchasing power. In this light, the poverty alleviation programme should be encouraged as a laudable programme not only for economic empowerment but also for improving health. Health programmes should also be incorporated into the poverty alleviation programme.

Finally, the health insurance scheme would do well to insulate the Nigerian public on these high costs of the antiretroviral drugs.

\section{REFERENCES}

Adler, Michael W.: ABC of AIDS $5^{\text {th }}$ ed. BMJ Book Publishing Group, London, Table 1.1, p.1-2-3 (2001).

Adrian, Mindel and Melinda, Tenant-Flowers: Natural History and Managemente of Early HIV. BMJ Book Publishing Group, London, P. 17 (2001).

Alubo, O., Best, E.G. and Best, K.: Report of the LATH
Desk Research on STD/HIV. Management Project in Nigerian, Jos, Nigeria (2000).

Christopher Haslett, Chivers E.R., Hunter, J.A.A. and Boon, N. A. (Eds.): Davidson's Principles and Practice of Medicine: A Textbook for Students and Doctors (18th Ed) Churchill Livingstone Edinburgh, Table 2.12, p. 92 (1999).

Cohen, John: AIDS, the unanswered questions of science. Science, 260: 1257 (1993).

Federal Ministry of Health: Summary Findings for the HIV/SYPHILIS Sentinel Sero-prevalence Survey in Nigeria. Abuja, Nigeria (1999).

HEAP: HIV/AIDS Emergency Action Plan. Developed by NACA in cooperation with USAID, FMOH, Abuja, Nigeria (2001).

National AIDS and STD Control Programme 2001. The 2001 National HIV/Syphilis Sentinel Survey among Pregnant Women Attending Ante-natal Clinics in Nigeria National AIDS/STDs Control Programme, The Federal Ministry of Health; Department of Public Health, Abuja, Nigeria, December, Technical report.

Tamen, F. and Udzua, F.: An Institutional Survey of Organizations which Support Rural Communities in the Livelihood Development in Mbashoho communities and Ushongo LGA. CEC/IFPR-DFID (2000).

Weller, I.V.D and Williams I.G.: $A B C$ of AIDS:Treatment of infections. British Medical Journal, 322: 13501354 (2001) 